\title{
A retrospective study of single frozen-thawed blastocyst transfer
}

\author{
Yong Soo Hur', Eun Kyung Ryu', Seung Hyun Song', San Hyun Yoon², Kyung Sil Lim', Won Don Lee', Jin Ho Lim \\ 'Maria Fertility Hospital, Seoul; ${ }^{2}$ Maria Research Center, Seoul, Korea
}

Objective: To study the clinical outcomes of single frozen-thawed blastocyst transfer cycles according to the hatching status of frozen-thawed blastocysts.

Methods: Frozen-thawed blastocysts were divided into three groups according to their hatching status as follows: less-than-expanded blastocyst ( $\leq \mathrm{EdB})$, hatching blastocyst $(\mathrm{HgB})$, and hatched blastocyst $(\mathrm{HdB})$. The female age and infertility factors of each group were evaluated. The quality of the single frozen-thawed blastocyst was also graded as grade A, tightly packed inner cell mass (ICM) and many cells organized in the trophectoderm epithelium (TE); grade B, several and loose ICM and TE; and grade C, very few ICM and a few cells in the TE. The clinical pregnancy and implantation rate were compared between each group. The data were analyzed by either $t$-test or chi-square analysis.

Results: There were no statistically significant differences in average female ages, infertility factors, or the distribution of blastocyst grades A, B, and $\mathrm{C}$ in each group. There was no significant difference in the clinical pregnancy and implantation rate of each group according to their blastocyst grade. However, there was a significant difference in the clinical pregnancy and implantation rate between each group. In the HdB group, the clinical pregnancy and implantation rate were similar regardless of the blastocyst quality.

Conclusion: There was an effect on the clinical outcomes depending on whether the blastocyst hatched during single frozen-thawed blastocyst transfer. When performing single frozen-thawed blastocyst transfer, the hatching status of the frozen-thawed blastocyst may be a more important parameter for clinical outcomes than the quality of the frozen-thawed blastocyst.

Keywords: Blastocyst; Single embryo transfer; Vitrification

\section{Introduction}

During in vitro fertilization (IVF), embryo cryopreservation is useful for surplus embryos after IVF cycles and provides an opportunity to perform an additional embryo transfer without a full IVF cycle [1]. Blastocyst transfer produces better clinical results than cleavage stage embryo transfer because of better embryo selection, an increased implantation rate, and an increased rate of pregnancy [2-6]. In recent years, for both fresh IVF cycles and frozen-thawed cycles,

Received: Sep 17, 2015 · Revised: Nov 24, 2015 · Accepted: Jan 30, 2016 Corresponding author: Yong Soo Hur

Maria Fertility Hospital, 20 Cheonho-daero, Dongdaemun-gu, Seoul 02586, Korea

Tel: +82-2-2250-5572 Fax:+82-2-2250-5585 E-mail:geaher@mariababy.com

This is an Open Access article distributed under the terms of the Creative Commons Attribution Non-Commercial License (http://creativecommons.org/licenses/by-nc/4.0/) which permits unrestricted non-commercial use, distribution, and reproduction in any medium, provided the original work is properly cited. single embryo transfer has been an important issue. Frozen-thawed blastocysts share potential advantages with fresh blastocysts. For example, frozen-thawed blastocyst transfer reportedly has a superior pregnancy rate, even though only one or two blastocysts are transferred [7-9]. In this approach, blastocysts are cryopreserved on day 5 or 6 from fresh cycles in which surplus embryos remained. The morphology of the blastocysts on these days varies from early blastocysts to hatched blastocysts. The majority of the blastocyst grading system is based on the degree of blastocoel expansion, the quality of the inner cell mass (ICM), and the appearance of the trophectoderm epithelium (TE) [10]. Briefly, the ICM is graded as A (tightly packed, many cells), B (loosely grouped, several cells), and C (very few cells), and the TE is graded as A (many cells forming a cohesive epithelium), $B$ (few cells forming a loose epithelium), and C (very few large cells). However, within such a grading system, there has been little analysis of the hatching status of the blastocyst. In case of thawing on the day be- 
fore transfer, as is routinely performed, over $70 \%$ of frozen-thawed blastocysts are in the hatching or hatched status on the day of transfer. Thus, a complement to the blastocyst grading system is needed that considers the hatching status.

The morphology prior to freezing is an important factor used to predict embryo viability $[11,12]$. However, the viability of a frozenthawed blastocyst after transfer remains unknown and the prefreeze blastocyst grading system is not a suitable indicator for frozenthawed blastocysts because of a lack of analysis of the hatching pattern, as previously discussed. Therefore, it is necessary to determine the differences in clinical outcomes, not only for the quality of the blastocyst but also for the form of the frozen-thawed blastocyst. Several researchers reported that the combination of pre-freeze and post-thaw morphological parameters could be used to predict live birth outcomes after frozen-thawed blastocyst transfer cycles, and the timing of the post-thaw blastocyst transfer could also be used to predict implantation $[13,14]$.

The objective of this study was to study the clinical outcomes of single frozen-thawed blastocyst transfer cycles according to the hatching status of frozen-thawed blastocysts.

\section{Methods}

\section{Patients, stimulation and embryo culture}

A total of 412 cycles of single frozen-thawed transfer cycles performed at the Maria Fertility Hospital from January 2011 to December 2014 were retrospectively analyzed. Female age, causes of infertility, and the number of prior pregnancies were assessed. The causes of infertility before frozen-thawed blastocyst transfer included female factors, male factors, combined factors, and unexplained infertility. Gonadotropin-releasing hormone $(\mathrm{GnRH})$ agonist long protocol or $\mathrm{GnRH}$ antagonist protocol was used in fresh cycles. In the $\mathrm{GnRH}$ agonist long protocol, patients were down-regulated using $\mathrm{GnRH}$ agonist (Superfact, Sanofi-Aventis, Frankfurt, Germany) and stimulation with recombinant follicle stimulating hormone (Gonal F, Merck Serono, Darmstadt, Germany) was started on the third day of menstruation. In $\mathrm{GnRH}$ antagonist protocol, ovarian stimulation was started on the third day of menstruation. GnRH antagonist was administered when the leading follicular size is $14 \mathrm{~mm}$ or more. When two or more follicles reached $18 \mathrm{~mm}$ in diameter, a dose of 10,000 IU human chorionic gonadotropin (hCG; Ovidrel, Merck Serono) was administered in both protocols.

Oocyte retrieval was performed 36 hours after hCG injection. Fertilization was assessed 15 to 18 hours after insemination based on the presence of two pronuclei. The zygotes were washed and cultured in groups of less than 5 in a $50 \mu \mathrm{L}$ micro-droplet (Sydney IVF Cleavage Medium-CM, COOK, Brisbane, Australia) for 48 hours, and the embry- os were subsequently selected for transfer. Some surplus embryos were cultured in a $50 \mu \mathrm{L}$ micro-droplet (Sydney IVF Blastocyst Medium-BM, COOK) for blastocyst development, and the others were cryopreserved or discarded. All culturing of the embryos was performed in a $\mathrm{CO}_{2}: \mathrm{O}_{2}: \mathrm{N}_{2}$ (6\%:5\%:89\%) environment.

\section{Pre-freezing blastocyst morphology}

Based on the report by Gardner et al. [15], we graded the blastocyst prior to transfer as A, B, or C according to the degree of blastocoel expansion, the quality of the ICM development, TE appearance and degree of necrosis [16]. The degree of blastocoel expansion was not the important consideration since cryopreservation was performed in the middle expanding stage or afterwards. Therefore, blastocysts were classified mainly by the quality of the ICM and TE appearance. Grade A was numerous tightly packed cells in the ICM and many TE cells organized in the epithelium. Grade B was several loosely packed cells in the ICM and several TE cells in the loose epithelium. Grade C was very few cells in the ICM and few large TE cells in the epithelium.

\section{Vitrification and thawing}

The vitrification was performed on blastocysts obtained from fresh cycles in which surplus embryos remained on day 5 or 6 . The blastocysts were equilibrated in Dulbecco's phosphate-buffered saline (DPBS, Thermo, Carlsbad, CA, USA) supplemented with 20\% serum substitute supplement (SSS; Irvine Scientific, Santa Ana, CA, USA) and 20\% ethylene glycol (EG; Sigma, Palo Alto, CA, USA) for $45 \mathrm{sec}$ onds. Then, the equilibrated blastocysts were moved into the vitrification solution consisting of 40\% EG, 18\% Ficoll (Sigma, Saint Louis, MO, USA) and 0.3 M sucrose (Sigma) dissolved in DPBS supplemented with $20 \%$ SSS for 20 seconds. After exposure, 1 or 2 blastocysts were quickly loaded into a thin plastic strip (TPS; SPL Life Science, Seoul, Korea) and plunged into liquid nitrogen $[17,18]$. All steps were performed at room temperature.

Embryos loaded on TPS were transferred for 5 minutes to a warming solution containing $0.5 \mathrm{M}$ sucrose dissolved in DPBS supplemented with $20 \%$ SSS followed by transfer to a second warming solution containing $0.125 \mathrm{M}$ sucrose dissolved in DPBS supplemented with $20 \%$ SSS for 5 minutes [19]. All steps were performed at room temperature. The embryos were then washed three times in MRC\#46 medium (Biosupply, Seoul, Korea) and cultured in an inner-well dish for further culture at $37^{\circ} \mathrm{C}$ in an atmosphere of $6 \% \mathrm{CO}_{2}, 5 \% \mathrm{O}_{2}$ and $89 \% \mathrm{~N}_{2}$. The post-thawing survival of the embryos was observed under an inverted microscope 16 to 20 hours after warming.

\section{Post-thaw blastocyst morphology}

Post-thaw blastocysts were also graded using the same reference as Gardner et al. [15]. We graded the blastocyst prior to transfer as A, 
$\mathrm{B}$, or $\mathrm{C}$ by combining the quality of the ICM development, TE appearance and degree of necrosis. Blastocyst morphology prior to transfer was also divided into three groups according to the hatching status. The less-than-expanded blastocyst ( $\leq \mathrm{EdB}$ ) group included early, middle expanded, expanded and fully expanded blastocysts, but not the hatching form. The hatching blastocyst $(\mathrm{HgB})$ group included hatching blastocysts, but not hatched blastocysts. The hatched blastocyst (HdB) group included completely hatched blastocysts.

\section{Single blastocyst transfer}

In the normal ovulation patients, the endometrium preparation protocol was based on detection of ovulation during a natural cycle. Estrogen, progesterone and luteal hormone were monitored. Some of the normal ovulation patients were administered hCG for accurate synchronization of the endometrium. In patients with irregular cycles, 2.5 to 5 mg letrozole (Femara, Novartis, Basel, Switzerland) was used for endometrium preparation on cycle day 3 . Then, follicle growth was monitored by ultrasound. When the follicles reached the criteria of maturation, hCG (10,000 IU) was injected to trigger ovulation. Hormone replacement therapy was also used for endometrial preparation. Two weeks after menstruation, $10 \mathrm{mg}$ of estrogen (estradiol-depot, EVER Pharma Jena GmbH, Jena, Germany) was given. When the endometrium thickness was greater than $8 \mathrm{~mm}$, progesterone (Taiyu progesterone, Taiyu Chemical \& Pharmaceutical, Taiwan) was started. The time of thawing embryos and transfer day was determined accordingly.

On the morning of embryo transfer, the thawed blastocysts were evaluated prior to transfer. Pregnancy was assessed by serum hCG concentration at 14 days post embryo transfer. Implantation was confirmed by the presence of a gestational sac. Clinical pregnancy was confirmed by the presence of fetal heart activity at 6 to 8 weeks in pregnancy.

\section{Statistics}

The data were examined using $t$-test or chi-square analysis to determine whether the differences in implantation and pregnancy rates were significant for each group. The results were considered statistically significant at $p$-values of $<0.05$.

\section{Results}

The $\leq \mathrm{EdB}, \mathrm{HgB}$, and $\mathrm{HdB}$ groups involved 103, 164, and 145 cycles, respectively. There was no significant difference in the average female age of the patients between the groups or differences in the ratio between infertility factors in each group. There was no significant difference in previous pregnancy history between each group. There was no significant difference in blastocyst grade $A, B$, and $C$ between each group (Table 1).

The clinical pregnancy and implantation rates of each group are summarized in Table 2. The implantation rate of the $\leq \mathrm{EdB}, \mathrm{HgB}$, and $\mathrm{HdB}$ groups were $8.7 \%, 22.0 \%$, and $47.6 \%$, respectively. The clinical pregnancy rate of the $\leq \mathrm{EdB}, \mathrm{HgB}$, and $\mathrm{HdB}$ groups were $8.7 \%$, $20.1 \%$, and $44.8 \%$, respectively. There was a significant improvement in the clinical pregnancy and implantation rates in the group transferred with hatched blastocysts.

The clinical pregnancy and implantation rates were compared in each group according to the blastocyst quality (Table 3). The average female age of each grade of blastocyst in each group was not significantly different. There was no significant difference in the clinical pregnancy rate of each group according to their blastocyst grade. The clinical pregnancy rate of the $B$ and $C$ grades was $12.5 \%$ and $8.4 \%$ in $\leq$ EdB group, respectively. In the $\mathrm{HgB}$ group, the clinical pregnancy rate of the $A, B$, and $C$ grades was $20.0 \%, 24.6 \%$, and $17.1 \%$, respectively. In the $\mathrm{HdB}$ group, the clinical pregnancy rate of the $A, B$, and $C$ grades was $46.2 \%, 47.4 \%$, and $42.7 \%$, respectively.

Table 1. Comparison of patients characteristics of the $\leq \mathrm{EdB}, \mathrm{HgB}$, and $\mathrm{HdB}$ groups

\begin{tabular}{lcccc}
\hline Patient characteristics & $\leq \mathrm{EdB}$ & $\mathrm{HgB}$ & $\mathrm{HdB}$ & 145 \\
\hline No. of patients & 103 & 164 & & \\
Female age $(\mathrm{yr})$ & & & $35.9 \pm 0.3$ & 0.2089 \\
$\quad$ Mean \pm SE & $36.1 \pm 0.4$ & $36.7 \pm 0.3$ & $27-48$ & 53 \\
$\quad$ Range & $29-46$ & $29-46$ & 25 & 0.6669 \\
Cause of infertility & 40 & 55 & 8 & 0.6087 \\
$\quad$ Female factor & 13 & 25 & 59 & 0.3809 \\
Male factor & 8 & 68 & $85(58.6)$ & 0.9889 \\
Combined factor & 42 & $110(67.1)$ & 0.2695
\end{tabular}

Values are presented as number or number (\%) unless otherwise indicated.

$\leq$ EdB group, included early, middle expanded, expanded and fully expanded blastocysts, but not the hatching form; HgB group, included hatching blastocysts, but not hatched blastocysts; $\mathrm{HdB}$ group, included completely hatched blastocysts; $\mathrm{SE}$, standard error of the mean. 
Table 2. Comparison of clinical outcomes of the $\leq \mathrm{EdB}, \mathrm{HgB}$, and $\mathrm{HdB}$ groups

\begin{tabular}{lcccc}
\hline Clinical outcomes & $\leq \mathrm{EdB}$ & $\mathrm{HgB}$ & $\mathrm{HdB}$ & \\
\hline Transferred cycles & 103 & 164 & 145 & - \\
Chemical pregnancy & $13(12.6)$ & $41(25.0)$ & $85(58.6)$ & 0.001 \\
Implantation & $9(8.7)$ & $36(22.0)$ & $69(47.6)$ & 0.001 \\
Clinical pregnancy & $9(8.7)$ & $33(20.1)$ & $65(44.8)$ & 0.001 \\
\hline
\end{tabular}

Values are presented as number or number (\%).

$\leq \mathrm{EdB}$ group, included early, middle expanded, expanded and fully expanded blastocysts, but not the hatching form; HgB group, included hatching blastocysts, but not hatched blastocysts; HdB group, included completely hatched blastocysts.

Table 3. Comparison of clinical outcomes of the $\leq \mathrm{EdB}, \mathrm{HgB}$, and $\mathrm{HdB}$ groups according to blastocyst quality

\begin{tabular}{|c|c|c|c|c|c|c|c|c|c|c|}
\hline \multirow{2}{*}{ Clinical outcomes } & \multicolumn{3}{|c|}{$\leq \mathrm{EdB}$} & \multicolumn{3}{|c|}{$\mathrm{HgB}$} & \multicolumn{3}{|c|}{$\mathrm{HdB}$} & \multirow{2}{*}{$p$-value } \\
\hline & Grade A & Grade B & Grade C & Grade A & Grade B & Grade C & Grade A & Grade B & Grade C & \\
\hline Female age (yr) & - & $36.8 \pm 1.0$ & $36.1 \pm 0.4$ & $35.6 \pm 1.2$ & $36.9 \pm 0.4$ & $36.5 \pm 0.4$ & $34.5 \pm 1.2$ & $35.7 \pm 0.5$ & $36.3 \pm 0.4$ & 0.415 \\
\hline Transferred cycle & - & 8 & 95 & 5 & 65 & 94 & 13 & 57 & 75 & - \\
\hline Chemical pregnancy & - & $1(12.5)$ & $12(12.6)$ & $1(20.0)$ & $20(30.8)$ & $20(21.3)$ & $8(61.5)$ & $36(63.2)$ & $41(54.6)$ & 0.001 \\
\hline Implantation & - & $1(12.5)$ & $8(8.4)$ & $1(20.0)$ & $17(26.2)$ & $18(19.1)$ & $6(46.2)$ & $28(49.1)$ & $35(46.7)$ & 0.001 \\
\hline Clinical pregnancy & - & $1(12.5)$ & $8(8.4)$ & $1(20.0)$ & $16(24.6)$ & $16(17)$ & $6(46.2)$ & $27(47.4)$ & $32(42.7)$ & 0.001 \\
\hline
\end{tabular}

Values are presented as mean $\pm \mathrm{SE}$ or number $(\%)$.

$\leq \mathrm{EdB}$ group, included early, middle expanded, expanded and fully expanded blastocysts, but not the hatching form; HgB group, included hatching blastocysts, but not hatched blastocysts; HdB group, included completely hatched blastocysts; SE, standard error of the mean .

However, there was a significant difference in the clinical pregnancy rate between each group. The clinical pregnancy rate of the $\mathrm{HgB}$ group was higher than the $\leq \mathrm{EdB}$ group, and that of the $\mathrm{HdB}$ group was higher than the $\mathrm{HgB}$ group. The pregnancy rate in the $\mathrm{HdB}$ groups was similar regardless of the blastocyst quality. There was no significant difference in the implantation rate of each group according to their blastocyst grade. However, there was a significant difference in the implantation rate between each group (Table 3).

\section{Discussion}

As the technology of preimplantation embryo culture has gradually developed, blastocyst transfer has also been increasing continuously. Generally, because of improved embryo selection, blastocyst transfer is advantageous for successful implantation and pregnancy rate compared to cleavage stage embryo transfer. As blastocyst transfer becomes generalized, there are continuing efforts to transfer a single embryo. Frozen-thawed blastocyst transfer has changed towards protocols that transfer a single embryo. The clinical outcomes of frozen-thawed embryo transfer depend on the quality of the preimplantation embryo before freezing or after thawing [20]. This study explored other parameters in addition to blastocyst quality that can predict the viability of frozen-thawed blastocysts after transfer. In this study, whether single frozen-thawed blastocysts were hatched prior to transfer had a significant impact on pregnancy. The clinical pregnancy rate of the $\mathrm{HgB}$ group was higher than the $\leq \mathrm{EdB}$ group, and that of the $\mathrm{HdB}$ group was higher than the $\mathrm{HgB}$ group. Clinical pregnancy in the $\mathrm{HdB}$ group was not significantly affected by the quality of the hatched blastocysts; even low-grade blastocysts in the $\mathrm{HdB}$ group yielded a pregnancy rate that was higher than high-grade blastocysts of the $\leq \mathrm{EdB}$ and $\mathrm{HgB}$ groups. Therefore, it is necessary to determine carefully the hatching status when performing single frozen-thawed blastocyst transfers.

The blastocyst grading system is primarily based on the degree of blastocoel expansion, as well as the quality of the ICM and TE. However, this grading system overlooks the hatching status of the blastocyst. In particular, in case of thawing on the day before transfer, as routinely performed, over $70 \%$ of frozen-thawed blastocysts are in the hatching or hatched status on the day of transfer. So, when the frozen-thawed blastocysts are graded, the hatching status of the blastocysts should be considered as a parameter before transfer. The morphology was less varied when thawing was performed on the day of transfer. However, thawing the day before or the day of transfer reportedly has no effect on clinical pregnancy rates [21]. The results of this study support thawing on the day before transfer.

We previously reported a mechanical technique for shrinkage using two 29-gauge needles [22]. The hatching rate may be influenced by the formation of a large hole in the zona pellucida (ZP), particularly with 29-gauge needle artificial shrinkage. Partial dissection of the ZP of frozen-thawed human embryos may enhance blastocyst hatching, implantation, and pregnancy rates [23,24]. Spontaneous hatching or hatched blastocysts show higher clinical pregnancies, 
embryo implantation, and live birth rates [25]. The assisted hatching of frozen-thawed blastocysts at the day 3 cleavage stage or of cleavage stage embryos is an effective procedure that can increase the pregnancy rate of human IVF [26]. Therefore, based on previous studies, the hatching or hatched form of blastocysts using assisted hatching may improve the clinical pregnancy. However, the study of assisted hatching of frozen-thawed blastocysts has not yet been performed. Therefore, additional studies are necessary to determine the effect of assisted hatching on frozen-thawed blastocysts.

The most suitable time for the assisted hatching of a frozen-thawed blastocyst is when it shrinks. It is easy to perform assisted hatching because, immediately after thawing, the blastocyst appears separated between the ZP and shrunk cell mass. Because overnight warmed blastocysts feature almost expanded blastocysts or a hatching state, it is difficult to perform assisted hatching.

It is crucial to have confidence in frozen-thawed blastocyst viability for single embryo transfer to be successful. Single embryo transfer is the preferred method for reducing multiple pregnancies when IVF is performed. However, it is a priority to screen viable embryos even during single embryo transfer $[27,28]$. From this point of view, when transferring a single frozen-thawed blastocyst, it is important to determine the quality of that blastocyst. It is also important to ascertain whether hatching may be an important criterion for a frozen-thawed single blastocyst transfer.

\section{Conflict of interest}

No potential conflict of interest relevant to this article was reported.

\section{References}

1. Gardner DK, Schoolcraft WB, Wagley L, Schlenker K, Stevens J, Hesla J. A prospective randomized trial of blastocyst culture and transfer in in- vitro fertilization. Hum Reprod 1998;13:3434-40.

2. Alves da Motta EL, Alegretti JR, Baracat EC, Olive D, Serafini PC. High implantation and pregnancy rates with transfer of human blastocysts developed in preimplantation stage one and blastocyst media. Fertil Steril 1998;70:659-63.

3. Levitas E, Lunenfeld E, Har-Vardi I, Albotiano S, Sonin Y, Hackmon-Ram R, et al. Blastocyst-stage embryo transfer in patients who failed to conceive in three or more day 2-3 embryo transfer cycles: a prospective, randomized study. Fertil Steril 2004; 81:567-71.

4. Schwarzler P, Zech H, Auer M, Pfau K, Gobel G, Vanderzwalmen P, et al. Pregnancy outcome after blastocyst transfer as compared to early cleavage stage embryo transfer. Hum Reprod 2004; 19:2097-102.
5. Desai N, Goldfarb J. Examination of frozen cycles with replacement of a single thawed blastocyst. Reprod Biomed Online 2005;11:349-54.

6. Papanikolaou EG, Kolibianakis EM, Tournaye $H$, Venetis CA, Fate$\mathrm{mi} \mathrm{H}$, Tarlatzis B, et al. Live birth rates after transfer of equal number of blastocysts or cleavage-stage embryos in IVF: a systematic review and meta-analysis. Hum Reprod 2008;23:91-9.

7. Yanaihara A, Yorimitsu T, Motoyama H, Ohara M, Kawamura T. Clinical outcome of frozen blastocyst transfer; single vs. double transfer. J Assist Reprod Genet 2008;25:531-4.

8. Han AR, Park CW, Lee HS, Yang KM, Song IO, Koong MK. Blastocyst transfer in frozen-thawed cycles. Clin Exp Reprod Med 2012;39:114-7.

9. Ishihara O, Araki R, Kuwahara A, Itakura A, Saito H, Adamson GD. Impact of frozen-thawed single-blastocyst transfer on maternal and neonatal outcome: an analysis of 277,042 single-embryo transfer cycles from 2008 to 2010 in Japan. Fertil Steril 2014; 101:128-33.

10. Shapiro BS, Daneshmand ST, Restrepo H, Garner FC, Aguirre M, Hudson C. Matched-cohort comparison of single-embryo transfers in fresh and frozen-thawed embryo transfer cycles. Fertil Steril 2013;99:389-92.

11. Heo YS, Yoon SH, Yoon HG, Cho HG, Yoon HG, Lee SW, et al. The studies on the development of human blastocyst embryos in IVF-ET program 1: the development of human blastocyst embryos by co-culture with cumulus cells. Korean J Fertil Steril 1996;23:155-62.

12. Steinberg ML, Boulet S, Kissin D, Warner L, Jamieson DJ. Elective single embryo transfer trends and predictors of a good perinatal outcome: United States, 1999 to 2010. Fertil Steril 2013;99:193743.

13. Ahlstrom A, Westin C, Wikland M, Hardarson T. Prediction of live birth in frozen-thawed single blastocyst transfer cycles by prefreeze and post-thaw morphology. Hum Reprod 2013;28:1199209.

14. Okimura T, Kuwayama M, Segawa T, Takehara Y, Kato K, Kato O. Relations between the timing of transfer, expansion size and implantation ratesin frozen thawed single blastocyst transfer. Fertil Steril 2009;92(3 Suppl):S246.

15. Gardner DK, Lane M, Stevens J, Schlenker T, Schoolcraft WB. Blastocyst score affects implantation and pregnancy outcome: towards a single blastocyst transfer. Fertil Steril 2000;73:1155-8.

16. Chimote NM, Mehta-Chimote BN, Chimote NN. Trophectoderm gradation and time of transfer are robust factors governing selection of best single blastocyst for transfer to predict implantation and live births in IVF [abstract P-179]. Hum Reprod 2004; 29(Suppl 1):i191. 
17. Son WY, Yoon SH, Yoon HJ, Lee SM, Lim JH. Pregnancy outcome following transfer of human blastocysts vitrified on electron microscopy grids after induced collapse of the blastocoele. Hum Reprod 2003;18:137-9.

18. Lee SY, Jae BS, Lee SY, Son WY, Yoon SH, Lim JH. Optimization of a thawing method for human expanded blastocysts vitrified using EM-Grid after artificial shrinkage. Fertil Steril 2003;80 Suppl 3:147-8.

19. Ryu EK, Hur YS, Ann JY, Maeng JY, Park M, Park JH, et al. Vitrification of mouse embryos using the thin plastic strip method. Clin Exp Reprod Med 2012;39:153-60.

20. Goto S, Kadowaki T, Tanaka S, Hashimoto H, Kokeguchi S, Shiotani M. Prediction of pregnancy rate by blastocyst morphological score and age, based on 1,488 single frozen-thawed blastocyst transfer cycles. Fertil Steril 2011;95:948-52.

21. Li T, Wang X, Yue C, Fang C. Comparison of pregnant outcomes of thawing blastocyst one day before transfer or not. Fertil Steril 2013;100(3 Suppl):S286.

22. Tucker MJ, Cohen J, Massey JB, Mayer MP, Wiker SR, Wright G. Partial dissection of the zona pellucida of frozen-thawed human embryos may enhance blastocyst hatching, implantation, and pregnancy rates. Am J Obstet Gynecol 1991;165:341-4.
23. Chimote NM, Chimote NN, Nath NM, Mehta BN. Transfer of spontaneously hatching or hatched blastocyst yields better pregnancy rates than expanded blastocyst transfer. J Hum Reprod Sci 2013;6:183-8.

24. Kung FT, Lin YC, Tseng YJ, Huang FJ, Tsai MY, Chang SY. Transfer of frozen-thawed blastocysts that underwent quarter laser-assisted hatching at the day 3 cleaving stage before freezing. Fertil Steril 2003;79:893-9.

25. Ge HS, Zhou W, Zhang W, Lin JJ. Impact of assisted hatching on fresh and frozen-thawed embryo transfer cycles: a prospective, randomized study. Reprod Biomed Online 2008;16:589-96.

26. Hur YS, Park JH, Ryu EK, Yoon HJ, Yoon SH, Hur CY, et al. Effect of artificial shrinkage on clinical outcome in fresh blastocyst transfer cycles. Clin Exp Reprod Med 2011;38:87-92.

27. Roy TK, Bradley CK, Bowman MC, McArthur SJ. Single-embryo transfer of vitrified-warmed blastocysts yields equivalent livebirth rates and improved neonatal outcomes compared with fresh transfers. Fertil Steril 2014;101:1294-301.

28. Kang SM, Lee SW, Jeong HJ, Yoon SH, Koh MW, Lim JH, et al. Clinical outcomes of elective single morula embryo transfer versus elective single blastocyst embryo transfer in IVF-ET. J Assist Reprod Genet 2012;29:423-8. 